\title{
Generation of pseudo-radially-polarized beams in a diode- pumped solid-state laser
}

\author{
J. M. O. Daniel, W. A. Clarkson \\ Optoelectronics Research Centre, University of Southampton, UK \\ e-mail:jmodlg09@orc.soton.ac.uk
}

\begin{abstract}
A simple technique for directly generating pseudo-radially-polarized or pseudo azimuthallypolarized donut-shaped beams in a diode-pumped solid-state laser is presented.Preliminary results for a Nd:YAG laser are described and the further potential of this technique is considered.

OCIS codes: (140.3410) Laser resonators; (140.3300) Laser beam shaping;(260.5430) Polarization ;
\end{abstract}

\section{Introduction}

Techniques for efficiently generating Laguerre-Gaussian (LG) modes with a donut-shaped intensity profile have become an increasing important topic for research owing to applications in a wide range of areas, from the life sciences to materials processing [1]. Interest in part comes from the unique properties of these beams derived by the ability to select radial (or azimuthal) polarization states. Indeed, this feature has been strongly linked with the enhanced laser processing speeds and improved edge definition in a range of materials processing applications.

A number of different techniques for generating ring-shaped LG beams have been devised over the years. The most popular techniques for low power applications exploit external beam-shaping arrangements (e.g.[2,3]). However, for applications (e.g. laser processing) that require high power levels, direct excitation in a laser resonator is generally preferred as it offers a route to higher power. Nevertheless, selective excitation of a high quality, loworder ring mode $\left(\mathrm{LG}_{01}\right)$ with a radial (or azimuthal) polarization in a laser architecture that is compatible with operation at high power levels and with flexibility in mode of operation is still quite challenging. This can be explained by considering that a radially-polarized $\mathrm{LG}_{01}$ is a coherent superposition of linearly-polarized $\mathrm{TEM}_{01}$ and $\mathrm{TEM}_{10}$ Hermite-Gaussian modes with a $\pi / 2$ phase shift (as shown in fig.1). Thus, any departure from axial symmetry with respect to polarization (e.g. due to polarization-dependence of loss in a Q-switch) will degrade polarization purity and mode quality.

In this paper we describe an alternative approach for directly generating a donut-shaped laser beam with pseudo-radial (or pseudo-azimuthal) polarization. In our scheme, orthogonally-polarized $\mathrm{TEM}_{01}$ and $\mathrm{TEM}_{10}$ modes are simultaneously excited and incoherently-combined within the laser resonator to yield an output beam with identical near-field and far-field time-averaged intensity profiles to the Laguerre-Gaussian $\left(\mathrm{LG}_{01}\right)$. This approach was tested on a diode-pumped Nd:YAG laser as described below.
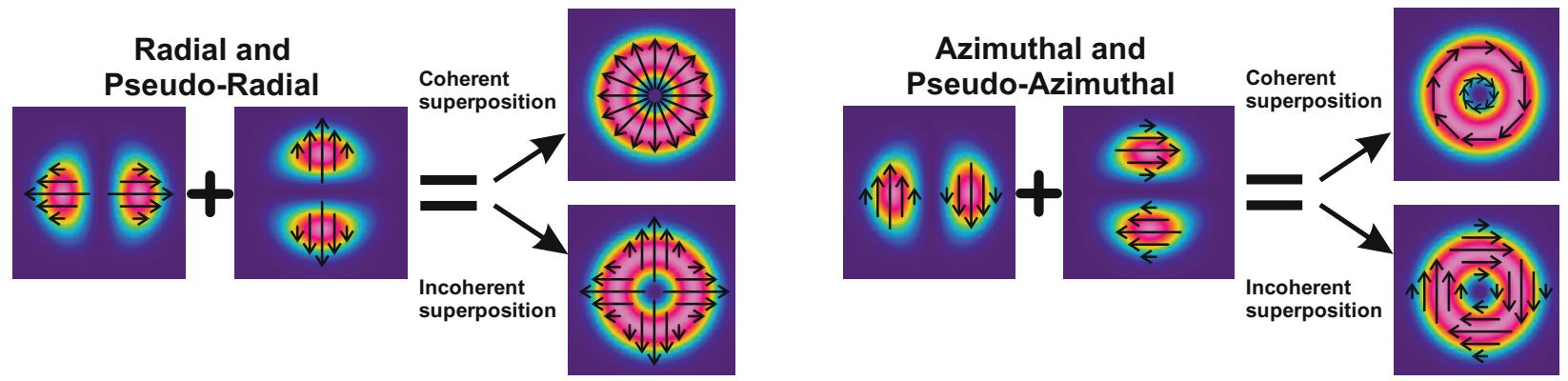

Fig. 1. Coherent and incoherent superposition of $\mathrm{TEM}_{01}$ and $\mathrm{TEM}_{10}$ beams to form donut-shaped beams.

\section{Experiment and results}

The experimental laser set-up is shown schematically in fig.2. A simple folded resonator design was employed comprising a plane pump incoupling and laser out-coupling mirror with high transmission at the pump wavelength $(808 \mathrm{~nm})$ and $3 \%$ transmission at the lasing wavelength $(1064 \mathrm{~nm})$, two curved high reflectors and a plane high reflector. A 4mm long Nd:YAG rod was used as the gain medium. The latter was mounted in a water-cooled copper heat-sink located in close proximity to the output coupler. Pump light was provided by a $4 \mathrm{~W}$ fiber-coupled diode laser at $808 \mathrm{~nm}$. The latter was re-shaped using capillary fiber with outer diameter of $200 \mu \mathrm{m}$ and an inner 
hole diameter of $100 \mu \mathrm{m}$ to provide a ring-shaped pump beam which was then spatially-matched to the $\mathrm{LG}_{01}$ mode profile in the Nd:YAG rod to provide preferential gain for the $\mathrm{TEM}_{01}$ and $\mathrm{TEM}_{10}$ modes. The capillary fiber was tapered over a length of $10 \mathrm{~mm}$ to an outer diameter of $\sim 105 \mu \mathrm{m}$ and spliced to the $105 \mu \mathrm{m}$ diameter multimode pump delivery fiber to simplify the pump beam transformation scheme and minimize the need for free-space optical components. Over $90 \%$ of the diode pump light was converted into the ring-shaped pump beam using this arrangement. A walk-off polarizer fabricated from BBO was also added to the cavity and positioned in close proximity to the final plane high reflector. The latter served to spatially separate orthogonally-polarized ordinary (o) and extraordinary (e) beams allowing selection of $\mathrm{TEM}_{01}$ and $\mathrm{TEM}_{10}$ modes with orthogonal linear polarization states using knife edge apertures at $90^{\circ}$ as shown in fig.2. Another important feature of this arrangement is the cavity lengths for the $\mathrm{o}$ and e beams are slightly different, so the resulting $\mathrm{TEM}_{01}$ and $\mathrm{TEM}_{10}$ have different axialmode frequencies and hence add incoherently. Pseudo-radial or pseudo-azimuthal polarization states can be selected depending on the orientation of the knife-edge apertures.

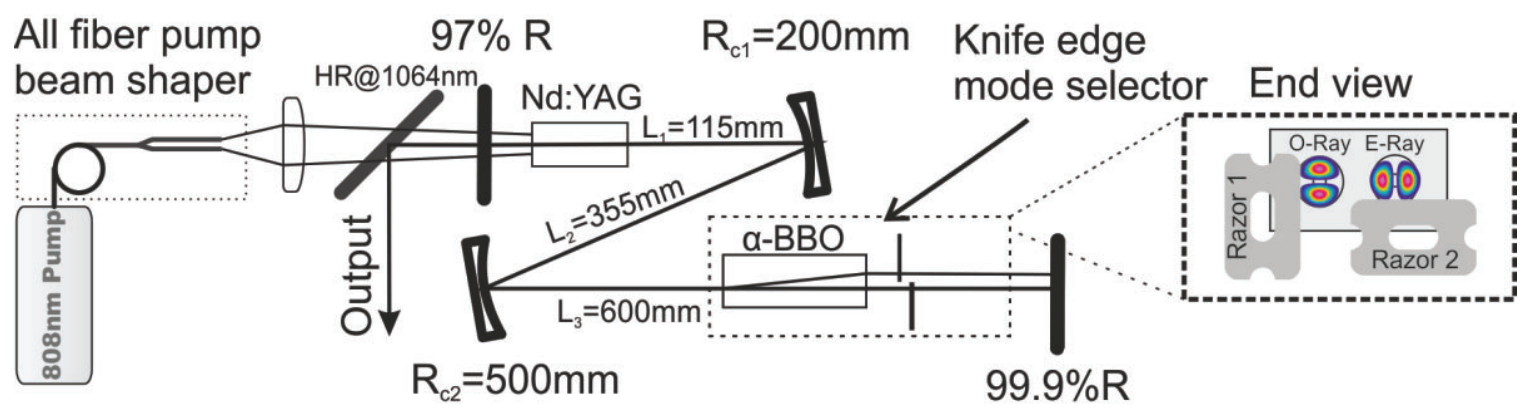

Fig. 2. Nd:YAG laser design

In both cases, the threshold pump power absorbed was $400 \mathrm{~mW}$ and the laser yielded a maximum output power of $500 \mathrm{~mW}$ with a corresponding slope efficiency of $30 \%$. The beam propagation factor $\left(\mathrm{M}^{2}\right)$ for the pseudo-radial mode was measured to be $\sim 2.2$ and hence very close to the theoretical value of 2 for an $\mathrm{LG}_{01}$ mode. Figure 3 shows the recorded intensity profiles for pseudo-radially polarized operation.

Fig. 1(a) shows output without a polarizer and figures (b) (c) and (d) show the transmission through a polarizer orientated at $0^{\circ}, 45^{\circ}$ and $90^{\circ}$ respectively. The results are in good agreement with expectations. Fig.3(c) highlights the difference between a pseudo-radial and a purely radially-polarised mode with the $45^{\circ}$ orientated polarizer resulting in a ring profile rather than a two lobe profile orientated at $45^{\circ}$ as would be expected for the true radially polarized LG mode case.
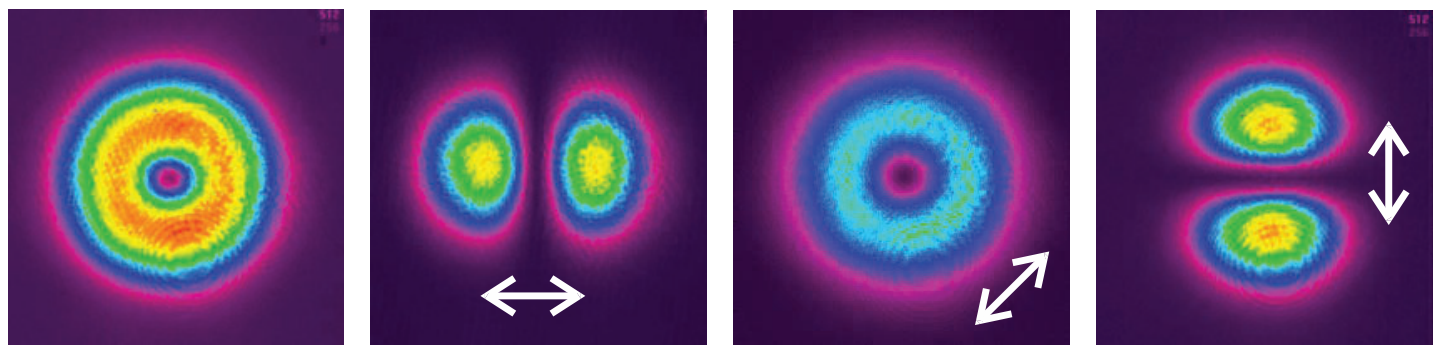

Fig. 1 Experimentally measured intensity profiles for a pseudo-radially-polarized beam (a) without polarizer (b) with polarizer at $0^{\circ}$, (c) with polarizer at $45^{\circ}$, (d) with polarizer at $90^{\circ}$.

\section{Conclusion}

In conclusion we have demonstrated a simple scheme for efficiently generating donut-shaped beams with pseudoradial (or pseudo-azimuthal) polarization. This approach is should be compatible with scaling laser power and should offer the flexibility required for extending mode of operation to the pulsed regime.

\section{References}

[1] Q. Zhan, "Cylindrical vector beams: from mathematical concepts to applications," Advances in Optics and Photonics, vol. 1, no. 1, p. 1, 2009.

[2] M. Stalder and M. Schadt, "Linearly polarized light with axial symmetry generated by liquid-crystal polarization converters.," Optics letters, vol. 21 , no. 23 , pp. 1948-50, Dec. 1996.

[3] G. Machavariani, Y. Lumer, I. Moshe, a Meir, and S. Jackel, "Efficient extracavity generation of radially and azimuthally polarized beams.," Optics letters, vol. 32, no. 11, pp. 1468-70, Jun. 2007. 